\title{
Planning of Relay Station Locations in IEEE 802.16 (WiMAX) Networks
}

\author{
Zakhia Abichar, Ahmed E. Kamal and J. Morris Chang \\ Department of Electrical and Computer Engineering \\ Iowa State University, Ames, IA 50011 \\ E-mail:\{abicharz, kamal, morris\}@iastate.edu
}

\begin{abstract}
Broadband wireless access networks have received tremendous attention in terms of research and development in the recent years. There have also been test networks deployed in many cities across the globe. In the IEEE 802.16j standard, relay stations (RS) play a promising role of extending the range of a base station (BS) especially in rural areas where it is difficult to install a large number of BSs with wired connections. Therefore, the planning of the number and location of relay stations is an important step in their deployment. In this paper, we address the problem of relay station planning in wireless broadband networks and we present an optimization for the RS planning problem in wireless broadband networks.
\end{abstract}

\section{INTRODUCTION}

Broadband wireless access networks have gathered a great momentum recently in terms of industry support, research and pilot network deployment. The group of companies supporting the development of the IEEE 802.16 family of standards, commercially known as the WiMAX, are members of the WiMAX Forum. The forum oversees the promotion of WiMAX through activities such as negotiating bandwidth licensing policies and authoring requirements of interoperability tests between equipment from various vendors. Research in wireless broadband networks has also picked up actively in many fields including resource allocation, relays placement, handover between cells, quality of service and others. Also, there are tens or even hundreds of pilot networks deployed around the globe to test drive the WiMAX technology.

This paper considers the problem of planning Relay Station (RS) locations in a wireless broadband access network, as in the architecture proposed in the IEEE 802.16j document [1]. A relay is considered as a stripped-down version of a base station (BS). While the BS has direct Internet connectivity, the RS does not. The RS is placed in the network to increase the connectivity between BSs and also to extend the coverage of a single BS. The RS does its functions in a cost-effective way since it only requires a power source to run, so it could be deployed easily in virtually any location. There are also systems that run relays (or base stations) on solar power [2], which makes the relays a good option for rural areas.

In this paper, we consider the problem of planning the locations of relays to extend the coverage of a single BS, as shown in Fig. 1. The location of the BS is given and it has wired connectivity. The RSs are used to extend the range of the BS without requiring further wired connectivity due to its higher expense especially in certain areas (such as rural areas) that are targeted by WiMAX. We could easily put more relays beyond the BS since they only require a power source to operate. In our problem, the location of the BS is given, while the locations of the RSs need to be determined to provide service to a given set of users with given demands in a given area. The objective of our work is to maximize the capacity of the network to transport the highest amount of data subject to meeting the demands of user's traffic, while minimizing the required infrastructure and covering the requested area of service. As a fairly reasonable assumption, we consider that the majority of the traffic originates from, or is destined to the BS; so traffic will be between the BS and the subscriber stations (SS) or mobile stations (MS). Also, there is traffic in the uplink and downlink directions and we consider that more traffic will go from the BS, across the relays, to the SSs or MSs; that is, the downlink traffic exceeds the uplink traffic. Therefore, the down links are the bottleneck and this is why we concentrate on them.

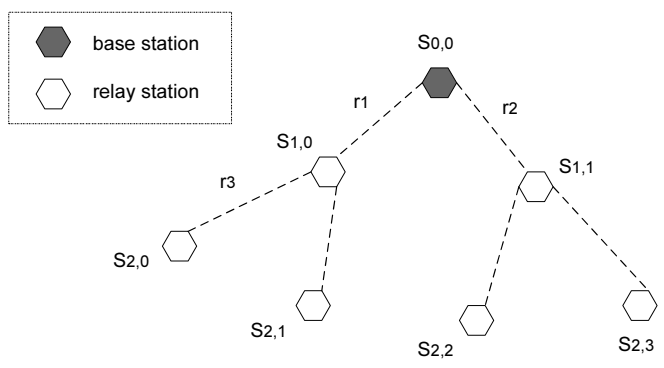

Fig. 1. BS with relays tree. $r_{i}$ are the rates of the links.

We formulate the problem into a mixed integer linear program (MILP). The optimization formulation produces the locations of the RSs and the rates on each link. The papers before us model the interference extensively. But they don't go into details on the capacity assignment. We do the link rate allocation that ensures the traffic will find enough bandwidth.

Finally, we present numerical results that show the usefulness of our model. We also show how our model can do the RS planning in a field with obstacles that would be present in real life scenarios.

The rest of this paper is organized as follows. Section II presents the related work and Section III introduces the network model of this paper. Section IV presents the optimization formulation of the problem and Section $\mathrm{V}$ gives some numerical results that illustrate the utility of our model. Finally, the 
paper concludes with summarizing remarks in Section VI.

\section{RELATED WORK}

A network planning model is presented in [3] to find the locations of BSs and RSs. The input of the model is a set of BS an RS potential locations and a set of Test Points (TPs) that represent the user data traffic. The planning problem is solved by optimization. The main limitation of this work is that the multihop links are limited to at most 2 hops from the BS. The considered topology is the following: the SS connects either to the BS, or to a RS that is connected to the BS. This assumption does not allow using the RS to its full potential in multihop links. In comparison, we allow RSs to be located several hops away from the BS, and be reachable through other RSs. The second limitation of this work is that they do not consider the link rate allocation and they do not consider users' requirements. They seek to minimize the power transmitted in the vicinity of a BS to reduce the interference and, hence, the number of users connected to a BS. But, they do not consider the maximum rate of BS-RS link and limit the number of connecting stations to the RS; however, we model link rate allocation in detail.

The same authors of the work above present an extension of their work in [4], where they consider a large coverage area that makes the computation time intractable. Thus, they divide the area into clusters and apply the approach above to every cluster. Then, they solve the cases on the boundaries of the clusters. The same limitation on the the maximum use of 2 hops in wireless still remains.

A related problem is the BS planning in UMTS systems. For example, a comparison of optimization solutions and algorithms for this problem is presented in [5]. The optimization formulation is mostly focused on modeling the interference from the users and aims to find the planning that reduces the interference. This part of the work is similar to [3], [4]. In addition, three heuristic-based algorithms are presented to find the BS sites. The results in this paper show that the heuristic algorithms provide an optimal solution in some cases (about half of the cases). Otherwise, they aren't far from the optimal solutions. Since the problem is in the context of UMTS, some parameters do not apply to the case of WiMAX. Also, in comparison, our model includes the rate allocation of links that is not part of this study.

A planning solution for WiMAX is presented in [6] where they allow the traffic of one SS to be allocated to several BSs. They also consider the capacity of the links and the requests of the TPs. However, only BS installation is considered and no RSs are included. The solution is a greedy algorithm that assigns the BS which connects to SS with lower degrees since those SS do not have many choices of BS connections.

Other approaches include [7], where the problem is deleting cells due to upgrading the network with more powerful BSs, which was solved by a heuristic-based algorithm.

None of the works above considers the multihop installation of RSs and modeling the capacity of the links at the same time. In our paper, we consider these two aspects in our model.
TABLE I

OFDMA RATES (IN MBPS) FOR VARIOUS MODULATION SCHEMES USING $7 \mathrm{MHZ}$ BANDWIDTH

\begin{tabular}{|c|c|c|c|c|c|}
\hline $\begin{array}{c}\text { QPSK } \\
1 / 2\end{array}$ & $\begin{array}{c}\text { QPSK } \\
3 / 4\end{array}$ & $\begin{array}{c}\text { 16-QAM } \\
1 / 2\end{array}$ & $\begin{array}{c}\text { 16-QAM } \\
3 / 4\end{array}$ & $\begin{array}{c}\text { 64-QAM } \\
2 / 3\end{array}$ & $\begin{array}{c}\text { 64-QAM } \\
3 / 4\end{array}$ \\
\hline 5.82 & 8.73 & 11.64 & 17.45 & 23.27 & 26.18 \\
\hline
\end{tabular}

\section{NETWORK MODEL}

This section presents the network model that we consider. First, we explain what characterizes the capacity of a link. Then, we state our assumption on channel assignment.

\section{A. Link Capacity}

The capacity of a wireless link can be modeled with the Shannon-Hartley equation [8]. The upperbound on the capacity is: $C=B \cdot \log _{2}\left(1+\frac{S}{N}\right)$, where $C$ is the capacity in bit/sec, $B$ is the channel bandwidth in $\mathrm{Hz}, S$ is the received signal power and $N$ is the noise ( $S / N$ is the signal-to-noise ratio or SNR). This equation indicates that the capacity changes with the distance since the SNR degrades for long distances due to path loss.

The other factors that affect the achievable rate over a channel are the coding and modulation schemes. With high SNR, the good channel quality is leveraged to achieve a high rate. With low SNR, robust schemes are used to counter the bad channel and limit the loss due to the high bit-error rate (BER). Hence, we express the maximum rate between nodes $i$ and $j$ by: $m_{i, j}=\Gamma\left(S N R_{i, j}, \beta\right.$, modulation $)$, where $\beta$ is the upperbound on the BER and $\Gamma$ is the function that maps all three parameters to the transmission rate. The standard [9] lists the achievable raw bit rates for the OFDMA physical layer as shown in Table I.

\section{B. Channel Assignment}

In the RS domain, we assume that two links interfering with each other are assigned different channels. In this way, the channel assignment can be solved using a tree coloring problem. Two nodes that connect to each other are connected by a link that is a assigned a "color". The link colors are reused within the graph to save bandwidth resources. We choose this assumption since it simplifies our work without loss of generality. Another approach is to have all of the RSs transmit in one channel and use time division multiplexing. In this paper, we do not address the scheduling scheme in detail since it is out of scope of this paper.

\section{OptIMIZATION}

In this section, we formulate the relay planning as an optimization problem. We start with the following definitions. Let $R=\{1, \ldots, n\}$ be the set of candidate sites for RS, and let $T=\{1, \ldots, m\}$ be the set of Test Points (TP) that designate the user traffic.

In our work, we assume that the majority of traffic is in the downlink direction. Hence, we present the solution based on the downlink only. Secondly, we assume that, for a link, 
the maximum flow is based on the power budget, distance, channel gain, and modulation scheme. For a given link length, multiple rates can be assigned on it with each one having its own bit-error-rate (BER). In this paper, for a given link length, we consider a rate with a certain BER, say $\beta$, and for every distance, we consider the corresponding rates that yields a BER that is less or equal to $\beta$. In this way, the multiple hops on a path have a comparable value of BER along the path.

\section{A. Decision Variables}

The decision variables of the optimization problem are the following:

$$
\begin{aligned}
D_{i}^{R} & = \begin{cases}1 ; & \text { an } \mathrm{RS} \text { is deployed in site } \mathrm{i}, i \in R \\
0 ; & \text { otherwise }\end{cases} \\
D_{i}^{B R} & = \begin{cases}1 ; & \mathrm{RS} i \text { is connected to the } \mathrm{BS}, i \in R \\
0 ; & \text { otherwise }\end{cases} \\
D_{i}^{B T} & = \begin{cases}1 ; & \mathrm{TP} i \text { is assigned to the } \mathrm{BS}, i \in T \\
0 ; & \text { otherwise }\end{cases} \\
D_{i j}^{R R} & = \begin{cases}1 ; & \mathrm{RS} i \text { is connected to } \mathrm{RS} j, i, j \in R \\
0 ; & \text { otherwise }\end{cases} \\
D_{i j}^{R T} & = \begin{cases}1 ; & \mathrm{TP} j \text { is assigned to } \mathrm{RS} i, i \in R, j \in T \\
0 ; & \text { otherwise }\end{cases}
\end{aligned}
$$

Secondly, for every link that is set in the problem, that is when $D_{i}^{B R}=D_{i}^{B T}=D_{i j}^{R R}=D_{i j}^{R T}=1$, we need to find the corresponding flow values, $f_{i}^{B R}, f_{i}^{B T}, f_{i j}^{R R}, f_{i j}^{R T}$ that have a positive real value. Thus, this makes our problem a mixedinteger linear problem (MILP).

\section{B. Topology Constraints}

The following constraints are applied to our problem. First, when a link between the BS and RS $i$ is assigned, we have to make sure that RS $i$ is indeed existent, hence we have the constraint:

$$
D_{i}^{B R} \leq D_{i}^{R} ; \quad \forall i \in R
$$

Similarly, when a link between $\mathrm{RS} i$ and $\mathrm{RS} j$ is assigned as existent, both of these RSs should be existent as well. Hence, we have:

$$
D_{i j}^{R R} \leq \frac{D_{i}^{R}+D_{j}^{R}}{2}
$$

In addition, when RS $i$ is assigned to TP $j$, the RS should be existent in the network. Hence,

$$
D_{i j}^{R T} \leq D_{i}^{R} ; \quad \forall i \in R, \forall j \in T
$$

We also need to add a constraint that limits the TP to connect to only the BS or, if not, one RS, using the following constraint.

$$
D_{i}^{B T}+\sum_{j \in R} D_{j i}^{R T}=1 ; \quad \forall i \in T
$$

\section{Flow Constraints}

In addition to the constraints above, we add the flow conservation constraints. These constraints ensure that (1) the total flow going out of the BS is sufficient for all the TP's traffic, (2) the flow going out of an RS is equal to all the flow going into the RS, and (3) the flow going into a TP is equal to the traffic of the TP. We define the rate matrices $f_{i}^{B R}, f_{i}^{B T}, f_{i j}^{R R}, f_{i j}^{R T}$ to be the flow rate from the BS to RS $i$, the rate from $\mathrm{BS}$ to $\mathrm{TP} i$, the rate from $\mathrm{RS} i$ to $\mathrm{RS} j$ and the rate from $\mathrm{RS} i$ to $\mathrm{TP} j$, respectively.

1) Flow Constraint at the BS: This constraint makes sure that the BS is giving out a total of capacity that is sufficient.

$$
\sum_{i \in R} f_{i}^{B R} \cdot D_{i}^{B R}+\sum_{j \in T} f_{j}^{B T} \cdot D_{j}^{B T}=\sum_{j \in T} r_{j}
$$

To keep the system linear, we use the following transforms:

$$
\begin{aligned}
& X_{i}=f_{i}^{B R} \cdot D_{i}^{B R} \quad \text { and, } \\
& Y_{j}=f_{j}^{B T} \cdot D_{j}^{B T}
\end{aligned}
$$

Hence, Eq. (10) becomes,

$$
\sum_{i \in R} X_{i}+\sum_{j \in T} Y_{j}=\sum_{j \in T} r_{j}
$$

which can be solved by the following constraints. Let $Q$ be a large number such that $Q>\max \left(f_{i}^{B R}, f_{j}^{B T}\right), i \in R, j \in T$.

$$
\begin{array}{ll}
X_{i} \geq Q \cdot D_{i}^{B R}-Q+f_{i}^{B R} & i \in R \\
X_{i} \leq f_{i}^{B R} & i \in R \\
X_{i} \geq 0 & i \in R \\
X_{i} \leq Q \cdot D_{i}^{B R} & i \in R \\
Y_{j} \geq Q \cdot D_{j}^{B T}-Q+f_{j}^{B T} & j \in T \\
Y_{j} \leq f_{j}^{B T} & j \in T \\
Y_{j} \geq 0 & j \in T \\
Y_{j} \leq Q \cdot D_{j}^{B T} & j \in T
\end{array}
$$

2) Flow Constraint at the RS: This constraint makes the incoming total flow at an RS equal to the outgoing total flow at the same RS, applied to all the RSs.

$f_{i}^{B R} \cdot D_{i}^{B R}+\sum_{j \in R} f_{j i}^{R R} \cdot D_{j i}^{R R}=\sum_{j \in R} f_{i j}^{R R} \cdot D_{i j}^{R R}+\sum_{k \in T} f_{i k}^{R T} \cdot D_{i k}^{R T}$

Similar to above, we replace $Z_{i j}=f_{i j}^{R R} . D_{i j}^{R R}, i, j \in R$ and $W_{i j}=f_{i j}^{R T} . D_{i j}^{R T}, i \in R, j \in T$ to make the system linear. Then, Eq. 22 becomes the following.

$$
X_{i}+\sum_{j \in R} Z_{j i}=\sum_{j \in R} Z_{i j}+\sum_{k \in T} W_{i k}
$$

We solve for $Z_{i j}$ and $W_{i j}$ as follows. 


$$
\begin{array}{rlrl}
Z_{i j} & \geq Q \cdot D_{i j}^{R R}-Q+f_{i j}^{R R} & i, j \in R \\
Z_{i j} \leq f_{i j}^{R R} & i, j \in R \\
Z_{i j} \geq 0 & i, j \in R \\
Z_{i j} \leq D_{i j}^{R R} \cdot Q & i, j \in R \\
W_{i j} \geq Q . D_{i j}^{R T}-Q+f_{i j}^{R T} & i \in R, j \in T \\
W_{i j} \leq f_{i j}^{R T} & i \in R, j \in T \\
W_{i j} \geq 0 & i \in R, j \in T \\
W_{i j} \leq Q . D_{i j}^{R T} & i \in R, j \in T
\end{array}
$$

3) Flow Constraint at the TP: This constraint ensures there is enough traffic flow for every TP.

$$
f_{i}^{B T} \cdot D_{i}^{B T}+\sum_{j \in R} f_{j i}^{R T} \cdot D_{j i}^{R T}=r_{i}
$$

We use the transforms above to make the equation linear as follows.

$$
Y_{i}+\sum_{j \in R} W_{j i}=r_{i}
$$

\section{Constraint on the Link Capacity}

The maximum values of the flow $f$ that can be transmitted on a link is limited by the following factors: the length of the distance, the transmit power and the coding and modulation schemes. We use the upperbound $m$ that specifies the maximum flow on a link. The constraints are as follows.:

$$
\begin{aligned}
f_{i}^{B R} \leq m_{i}^{B R} ; & \forall i \in R \\
f_{i}^{B T} \leq m_{i}^{B T} ; & \forall i \in T \\
f_{i j}^{R R} \leq m_{i j}^{R R} ; & \forall i, j \in R \\
f_{i j}^{R T} \leq m_{i j}^{R T} ; & \forall i \in R, \forall j \in T
\end{aligned}
$$

\section{E. Objective Function}

We need to minimize the total number of RSs that are used in the system such as to having the following objective function:

$$
\min \sum_{i} D_{i}^{R}
$$

\section{Numerical RESUlts}

This section presents examples that show how our model is used to plan a network. It also shows special real-life cases in the planning problem where there are obstacles in the coverage area.

\section{A. Planning}

We show a planning example that uses our model. The example is shown in Fig. 2. We assume that RSs can be located on the corners of a square grid shown on the left side of the figure. We assume a square grid shape. Thus, the input of the problem is the following: the side of the grid, the TPs, and the demand of TPs in Mbps. We place the TPs in the middle
TABLE II

LINK RATES USED IN OUR EXAMPLES

\begin{tabular}{|c|c|}
\hline Distance $($ unit $)$ & Link Rate $($ Mbps $)$ \\
\hline \hline if distance $<=1$ & rate $=10$ \\
\hline else if distance $<=2$ & rate $=5$ \\
\hline else if distance $<=3$ & rate $=2$ \\
\hline else if distance $<=4$ & rate $=1$ \\
\hline else & rate $=0$ \\
\hline
\end{tabular}

of the unit square, as in the figure. The solution gives us the number and placement of the RSs, the used links, and the rates of the links.

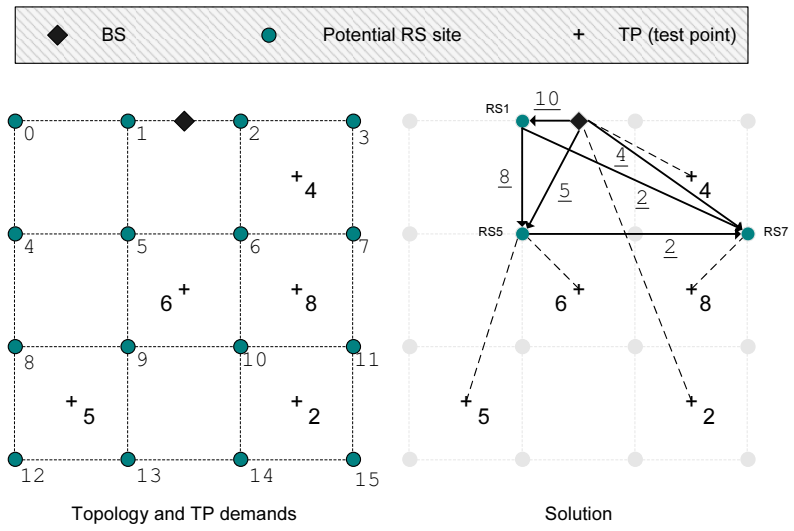

Fig. 2. Planning Example

In real life, there is a grounds survey which precedes a network deployment [10], [11], [12]. Based on the links characteristics (including SNR measurements and distance between the nodes), the maximum rates for every link are found $\left(m_{i}^{B R}, m_{j}^{B T}, m_{i, k}^{R R}, m_{i, j}^{R T}, i, k \in R, j \in T\right.$, from previous section). In our example, we use the rates that are summarized in Table II. The unit distance is the side length of a square in the grid. The rates in this example are based on the distance.

The solution to this planning example is shown on the right side of Fig. 2. Three RSs are needed for this problem which are the highlighted circles. They are RS1, RS5 and RS7. The solid line links are the BS-RS and RS-RS links. The dashed lines are the BS-TP and RS-TP links. The underlined numbers are the link rates allocated by the solution (subject to maximum link rate in Table II). The rates of the dotted links are equal to the corresponding TPs' rates, since in our solution the traffic of a TP is carried on a single link, either to an RS or the BS. We note the following observations from this example:

- The distance from the TP to the BS doesn't necessarily indicate a direct or relayed connection. For example, the TP with demand of 2 is the farthest from the BS. However, its demand is relatively low which can be satisfied by a single link. On the other hand, TPs which are closer to the BS have higher demands, and require the use of relay stations.

- Secondly, the RS to RS links help in reducing the number of relays. In our example, there is more traffic to the right of the BS $(4+8+2=14)$ than the left (5) and the middle 
(6). Thus, in the solution, the diagonal and horizontal links, both with rate of $2 \mathrm{Mbps}$, between RSs $(1,7)$ and $(5,7)$, respectively, relay the traffic from the right side to the less congested left side. If this was not the case, more relays would be needed on the right side.

\section{B. Obstacle Model}

In this part, we consider the existence of obstacles in the planning area. In real life, obstacles could be natural such as a hill, a forest or a lake, or man-made structures such as buildings, water towers or others.

In this subsection, we consider two cases of obstacles as shown in Fig. 3. First, there might be lakes or other similar obstacles in the area. This type of obstacles does not allow deploying an RS in it. However, two adjacent RSs can transmit on a link that goes over this types of obstacle, as shown in the figure. In the model, the RS location over this obstacle is canceled by setting the maximum rate on such RS to null. In the model, these are the parameters for when $R S_{i}$ is canceled: $m_{i}^{B R}=0$ as the rate to the $\mathrm{BS}, m_{i j}^{R R}=0, \forall j \in R$ as the rate to other RSs, and $m_{i k}^{R T}=0, \forall k \in T$ as the rate to all TPs.

The other type of obstacles that we consider doesn't allow a wireless link to penetrate it. For example, it could be a small hill in the area. We also consider that it might be rough terrain and it wouldn't allow deploying an RS on it. This type is also shown in Fig. 3. The same conditions that were for the first type apply here to cancel the use of RSs that coincide with the obstacle. In addition, we need to cancel the links that traverse the obstacle too. So for every link that is blocked by the obstacle from the BS to $R S_{i}$, from the $\mathrm{BS}$ to $T P_{j}$ or from $R S_{i}$ to $R S_{k}$ and from $R S_{i}$ to $T P_{j}$, we have $m_{i}^{B R}=$ $0, m_{j}^{B T}=0, m_{i k}^{R R}=0, m_{i j}^{R T}=0$.

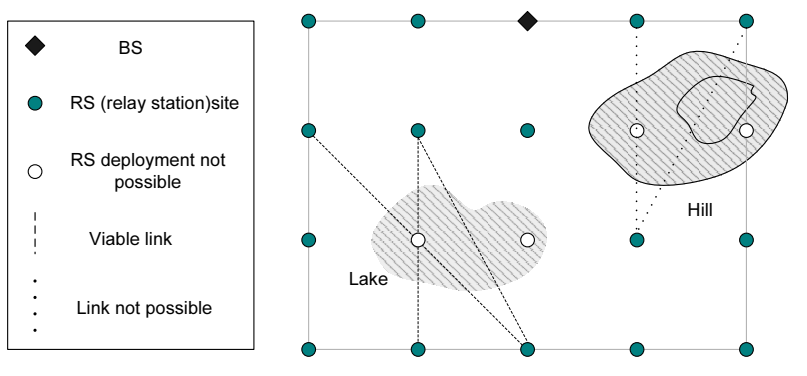

Fig. 3. Obstacle Model

\section{Planning with Lake-Type Obstacles}

Now we show how the planning is solved by our model with obstacles in the planning area. We take the planning case in Fig. 2 and we insert obstacles in it, once a lake and once a hill. Then we compare the planning outcome due to the effect of the obstacles.

First, we start by inserting a lake in the planning area as shown in Fig. 4. The lake covers the RS locations of RS5 and RS6. Hence, it is no longer possible to deploy RSs at these sites. We note the following observations in comparison to Fig. 2.

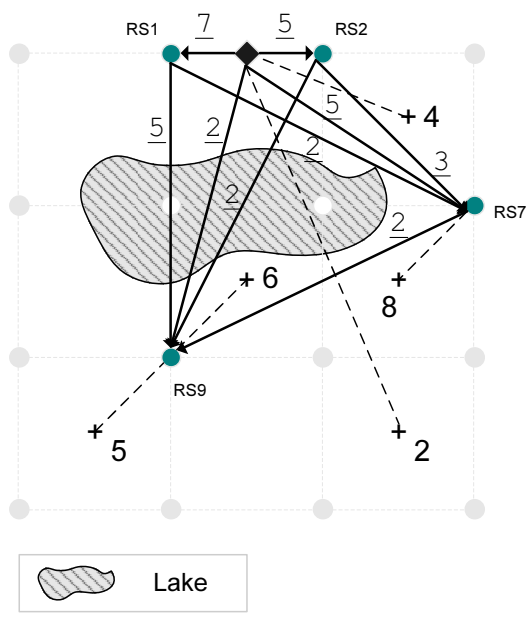

Fig. 4. Planning Result with Lake Obstacle

- The number of RSs has changed from 3 to 4 . This shows that an obstacle might increase the number of RSs. Previously, RS1, RS5 and RS7 were used. The obstacle makes RS5 no longer available. In the new planning, the RSs used are RSs 1, 2, 7 and 9.

- As we designated in the model, the links can traverse a lake-type obstacle. Hence, in the planning result, many links traversed the lake. Notably, from RS9, there are 3 links that go over the lake to RS1, the BS and RS2.

- Finally, the links that were direct from TPs to BS remain unchanged since the lake-type obstacle doesn't interfere with these links.

\section{Planning with Mountain-Type Obstacles}

In the following case, we insert a mountain-type obstacle in the planning area. This type of obstacle, as the lake-type, cancels the overlapping RS position. In addition, it cancels the links traversing it. Thus, the mountain-type obstacle is more restrictive than the lake-type. The result of the planning is shown in Fig. 5. The result shows that here, 5 RSs are now needed to support the TPs. This is intuitive since this obstacle has more restrictions than in the previous case, which required 4 RS, and compared to the initial case with no obstacles, which required 3 RSs.

We note the following observations:

- The network used links that are dispersed around the mountain, since it is not possible to go through it.

- Notice for TP4 (with rate of $2 \mathrm{Mbps}$ ), it was able to communicate directly with the BS in the case with no obstacles and in the case with lake obstacle. Now, it cannot do this anymore, because of the mountain, and it goes through RS2.

- Finally, we saw that more restrictions in the planning area on the RS locations and the viable links will likely require more RSs to satisfy the TPs.

\section{CONCLUSION}

This paper presented a planning model for Relay Stations (RS) deployment in WiMAX networks according to the IEEE 


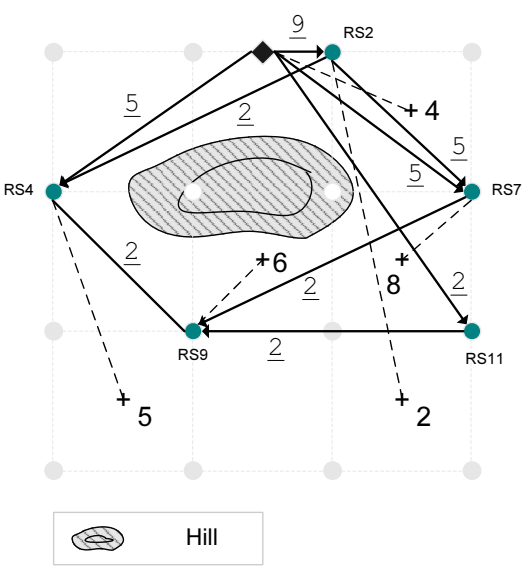

Fig. 5. Planning Result with Mountain Obstacle

802.16j standard. Our work is different from other proposed work in the literature in that it considers the conservation of flow on all the nodes. This provides for enough bandwidth for all the connections at every level of the RS grid. We formulated the problem into a mixed integer linear program that solves for the RS locations and the transmission rates on the links. The model takes as input the possible sites of RSs, the users demands for a number of TPs and minimizes the number of RSs used. We showed how our model can be used to plan a network of RSs. We also considered the existence of two types of obstacles in the planning area. The first is the laketype obstacle, and the second is a more restrictive type called mountain-type obstacles. We showed how our model can be used with each of the obstacle types. Finally, we provided observations and insight on the effect of obstacles on the planning results.

\section{REFERENCES}

[1] "Baseline Document for Draft Standard for Local and Metropolitan Area Networks Part 16: Air Interface for Fixed and Mobile Broadband Wireless Access Systems Multihop Relay Specification," IEEE 802.16j06/026r4, 2006.

[2] "A solar-powered wimax base station solution," Intel Application Note, Intel Netstructure(r) WiMax Baseband Card, http://www.intel.com/design/telecom/applnots/316021.pdf.

[3] Y. Yu, S. Murphy, and L. Murphy, "Planning base station and relay station locations in ieee 802.16j multi-hop relay networks," Consumer Communications and Networking Conference, 2008. CCNC 2008. 5th IEEE, pp. 922-926, Jan. 2008.

[4] _ - "A clustering approach to planning base station and relay station locations in ieee $802.16 \mathrm{j}$ multi-hop relay networks," Communications, 2008. ICC '08. IEEE International Conference on, pp. 2586-2591, May 2008.

[5] E. Amaldi, A. Capone, and F. Malucelli, "Planning UMTS Base Station Location: Optimization Models with Power Control and Algorithms," IEEE Transactions on Wireless Communications, vol. 2, no. 5, pp. 939952, 2003.

[6] P. Lin, H. Ngo, C. Qiao, X. Wang, T. Wang, and D. Qian, "Minimum cost wireless broadband overlay network planning," in World of Wireless, Mobile and Multimedia Networks, 2006. WoWMoM 2006. International Symposium on a, 2006, pp. 7 pp.-236.

[7] D. Abusch-Magder, "Novel algorithms for reducing cell sites during a technology upgrade and network overlay," in Wireless Communications and Networking Conference, 2005 IEEE, 2005.

[8] W. Stallings, Wireless Communications and Networks. Prentice Hall, 2001.

[9] "IEEE Standard for Local and Metropolitan Area Networks Part 16: Air Interface for Fixed Broadband Wireless Access Systems," IEEE Std 802.16-2004 (Revision of IEEE Std 802.16-2001), pp. 1-857, 2004.

[10] T. Theodoros and V. Kostantinos, "Wimax network planning and systems performance evaluation," in Wireless Communications and Networking Conference, 2007.WCNC 2007. IEEE, March 2007, pp. 1948-1953.

[11] M. Molina-Garcia and J. I. Alonso, "Planning and sizing tool for wimax networks," Radio and Wireless Symposium, 2007 IEEE, pp. 403-406, Jan. 2007.

[12] J. Garcia-Fragoso and G. Galvan-Tejada, "Cell planning based on the wimax standard for home access: a practical case," in Electrical and Electronics Engineering, 2005 2nd International Conference on, Sept. 2005, pp. 89-92. 